\title{
The powered vascular staple (PVS) versus conventional powered linier cutter (PLC) for the application of bronchial transection in thoracoscopic anatomic segmentectomy
}

\author{
Ying Ji", Bin Qiü, Shugeng Gao \\ Department of Thoracic Surgery, National Cancer Center/National Clinical Research Center for Cancer/Cancer Hospital, Chinese Academy of \\ Medical Sciences and Peking Union Medical College, Beijing 100021, China \\ Contributions: (I) Conception and design: B Qiu, Y Ji; (II) Administrative support: S Gao; (III) Provision of study materials or patients: B Qiu; \\ (IV) Collection and assembly of data: Y Ji; (V) Data analysis and interpretation: Y Ji; (VI) Manuscript writing: All authors; (VII) Final approval of \\ manuscript: All authors. \\ \#These authors contributed equally to this work. \\ Correspondence to: Shugeng Gao, MD. Department of Thoracic Surgery, National Cancer Center/National Clinical Research Center for Cancer/Cancer \\ Hospital, Chinese Academy of Medical Sciences and Peking Union Medical College, Beijing 100021, China. Email: gaoshugeng@vip.sina.com.
}

Background: Recent studies indicated that thoracoscopic anatomical segmentectomy (TAS) is widely adopted in early non-small cell lung cancer. Considering the slender segmental bronchi and the narrow space around segmental bronchi, it is easier to treat segmental bronchi with powered vascular stapler (PVS), which was designed to have a narrower anvil and a beak like tip. However, the safety and feasibility of PVS in stapling segmental bronchi are not yet clear.

Methods: We retrospectively compared the perioperative features of segmental bronchial closure treated with PVS and powered linier cutter (PLC) in thoracoscopic anatomic segmentectomy performed by a single surgeon and evaluated the safety and feasibility of PVS for the segmental or subsegmental bronchus.

Results: Between April 2018 and April 2019, a total of 162 patients who underwent anatomic segmentectomy were analyzed (PVS n=75 and PLC n=87). No bronchopleural fistula (BPF) occurred in 162 patients. Intraoperative blood loss in PVS group $(15.2 \pm 8.91 \mathrm{~mL})$ was significantly lower than that in PLC group $(25.29 \pm 24.06 \mathrm{~mL})(\mathrm{P}<0.05)$. The operative time of PVS group was similar to the PLC group $(\mathrm{P}>0.05)$. There was no significant difference between the two groups in postoperative drainage and chest tube duration (all $\mathrm{P}>0.05$ ). In terms of postoperative complications, the incidence of hemoptysis in PVS group was similar to PLC group $(\mathrm{P}>0.05)$. Two cases of subcutaneous emphysema occurred in PVS group, one case of pulmonary air leakage ( $>3$ days) and one case of subcutaneous emphysema occurred in PLC group. Besides, all the surgeons (100\%) slight agree or strongly agree that PVS is easier to pass through the segmental bronchus.

Conclusions: PVS is a smoother and easier instrument and has the same effectiveness and security as PLC. It leads to reduced operation difficulty and potential intraoperative complications.

Keywords: Powered vascular staple (PVS); powered linier cutter (PLC); thoracoscopic surgery; segmentectomy

Submitted Sep 24, 2019. Accepted for publication Oct 17, 2019.

doi: $10.21037 /$ jtd.2019.10.68

View this article at: http://dx.doi.org/10.21037/jtd.2019.10.68

(c) Journal of Thoracic Disease. All rights reserved. 


\section{Introduction}

With the increase of diagnoses of early lung cancer, more patients with stage I A non-small cell lung cancer benefit from early surgical treatment and obtain better longterm prognosis. Therefore, 'high quality' segmental or subsegmental resection based on anatomical lung segments has been reported and extensively studied $(1,2)$. Aokage et al. showed that segmentectomy, which could preserve the healthy lung tissue to the maximum extent, had the same prognosis as lobectomy in patients with nodules less than $2 \mathrm{~cm}$ (3). However, because of its more elaborate anatomical structure, segmentectomy puts forward higher requirements for surgeons' skill and endoscopy equipment.

In the development of minimally invasive thoracoscopic surgery, the improvement of endoscopic instruments plays a vital role. Stapling technique has been used in most thoracoscopic operations, including lung tissue incision, vascular transection and bronchial closure $(4,5)$. The use of vascular endostapler simplifies the procedure of pulmonary vessel transection, while the use of bronchial linier cutter reduces the occurrence of BPF to a large extent. The conventional powered linier cutter (PLC) can safely stapling the main bronchial trunk which average diameter is about $8.1 \mathrm{~mm}$ (6). However, the traditional bronchial transection devices are inconvenient to pass through segmental bronchi with narrow tissue space around it. Access to these segmental bronchi is challenging since they are easily mobile and located deep in the pulmonary tissue. Current endoscopic transection devices are not optimized to meet the unique challenges posed by the task of segmental bronchi transection. The anvil width of powered vascular stapler (PVS) is narrower than that of PLC, and the length of its staple is shorter than PLC, which makes PVS more precise placement on segmental bronchi. Besides, PVS has a $22^{\circ}$ upward tip, which was originally designed to enable more convenient pass through fragile pulmonary vessels, making it easier to access to tissue channels manoeuvrability. Japanese surgeons have reported the possibility of using PVS instead of PLC for the closure of the segmental bronchi, but its safety and feasibility still needs further verification (7).

Therefore, this study examined the safety and reliability of PVS in closing segmental bronchus by comparing the intraoperative and postoperative clinical indicators with PLC. In order to ensure the consistency of the operation, we selected the patients of a single operator to study. Furthermore, a subjective questionnaire was applied to
28 thoracic surgeons to assess the satisfaction of PVS in stapling segmental bronchi.

\section{Methods}

\section{Patient selection}

This is a single-surgeon, nonrandomized analysis on the feasibility of PVS in closing segmental bronchi. We retrospectively reviewed the clinical records of patients who underwent anatomic segmentectomy or subsegmentectomy at Department of Thoracic Surgery, National Cancer Center between April 2018 and April 2019. The inclusion criteria were: (I) patients selected for anatomical segmentectomy with the lesion $<3 \mathrm{~cm}$; (II) closure and transection of segmental bronchus with PVS or PLC. The exclusion criteria were conversion to thoracotomy or conversion to lobectomy. This study was approved by the institutional review board of Cancer Hospital, Chinese Academy of Medical Sciences.

\section{Surgical procedures and postoperative follow-up}

Patients were administered general anesthesia with doublelumen tracheal intubation, one-lung ventilation. The thoracoscopic anatomical segmentectomy (TAS) was performed in all patients via a three-port approach.

The treatment sequence of segmental bronchi, arteries, and veins was performed according to anatomical characteristics. Segmental arteries and veins were ligated with \#4 suture or transected with PVS, respectively. In PLC group, conventional ECHELON FLEX powered articulating endoscopic linear cutter (PSE45A; Ethicon Endo-Surgery, USA) was used to close the targeted segmental bronchi (Figure 1A). While in PVS group, targeted segmental bronchi were transected with ECHELON FLEX PVS (PVE35A; Ethicon Endo-Surgery, USA) (Figure 1B). "Inflation-deflation method" was generally used to ascertain intersegmental demarcation (8). The intersegmental plane was divided with a combination of electrocautery and stapler. In all cases, frozen sections were performed on nodule samples during operation, lymph nodes were sampled routinely. Systematic mediastinal lymph node dissection was performed in patients with invasive adenocarcinoma.

Patients were scheduled for outpatient review after 1 month [X ray or computed tomography CT)] and 3 months (CT). Postoperative fellow-up of patients 



Figure 1 The schemas of two powered devices for bronchial transection in thoracoscopic anatomic segmentectomy. (A) Closure of anterior segment bronchus of right upper lobe $\left(\mathrm{RB}^{3}\right)$ with powered linier cutter. (B) Powered vascular staple is applied to the left upper division subsegmental bronchus $\left(\mathrm{LB}^{1+2} \mathrm{c}\right)$.

focuses on severe complications occurred out-of-hospital. In our study, no severe complication was observed during outpatient follow-up.

\section{Statistical analysis}

SPSS 18.0 software (IBM Corporation, Armonk, NY, USA) was used to proofread the whole groups of data. Pearson Chi-square test or Fisher exact test were used to compare the categorical variables. The continuous variables were described by mean \pm standard deviation (SD) and $t$-test was performed. All $\mathrm{P}$ values were two sided with a significance level of 0.05 .
Table 1 Baseline of clinical characteristics

\begin{tabular}{|c|c|c|c|}
\hline Characteristics & PVS & PLC & $P$ value \\
\hline Gender, n (\%) & & & 0.157 \\
\hline Male & $19(25.3)$ & $31(35.6)$ & \\
\hline Female & $56(74.7)$ & $56(64.4)$ & \\
\hline Age (years) & $54.24 \pm 8.78$ & $54.64 \pm 9.04$ & 0.777 \\
\hline \multicolumn{4}{|l|}{$\begin{array}{l}\text { Pulmonary function, mean } \\
\% \text { pred }\end{array}$} \\
\hline DLCO & $98.10 \pm 25.26$ & $104.90 \pm 26.58$ & 0.117 \\
\hline FEV1 & $90.49 \pm 16.80$ & $86.64 \pm 18.83$ & 0.179 \\
\hline FVC & $89.75 \pm 14.82$ & $85.62 \pm 13.21$ & 0.065 \\
\hline Lesion diameter $(\mathrm{mm})^{\star}$ & $9.9 \pm 3.6$ & $10.6 \pm 3.5$ & 0.259 \\
\hline $\begin{array}{l}\text { Bronchial outer diameter } \\
(\mathrm{mm})\end{array}$ & $5.91 \pm 1.0$ & $6.11 \pm 0.88$ & 0.157 \\
\hline Bronchial wall thickness & $0.83 \pm 0.22$ & $0.89 \pm 0.24$ & 0.16 \\
\hline Pathologic type, n & & & 0.11 \\
\hline Benign nodule & 3 & 0 & \\
\hline AIS/MIA & 39 & 43 & \\
\hline $\begin{array}{l}\text { Other types of malignant } \\
\text { tumor }\end{array}$ & 38 & 46 & \\
\hline Comorbidity, $\mathrm{n}$ & & & 0.886 \\
\hline COPD & 2 & 2 & \\
\hline Diabetes mellitus & 5 & 8 & \\
\hline Hypertension & 20 & 19 & \\
\hline Heart diseases & 4 & 3 & \\
\hline
\end{tabular}

*, the largest dimension of lesion was measured for T category according to the 8th edition TNM staging. COPD, chronic obstructive pulmonary disease; \%pred, predicted value ratio; DLCO, diffusion capacity of the lung for carbon monoxide; FEV1, forced expiratory volume in 1 second; FVC, forced vital capacity; PVS, powered vascular stapler; PLC, powered linier cutter; AIS, adenocarcinoma in situ; MIA, minimal invasive adenocarcinoma.

\section{Results}

This study included 75 cases transected segmental bronchi with PVS and 87 cases transected with PLC. Data on age, gender, pulmonary function, maximum lesion diameter, pathological types, comorbidity, bronchial outer diameter (BOD) and bronchial wall thickness are summarized in Table 1. There was no significant difference in baseline characteristics between the two groups (all $\mathrm{P}>0.05$ ) (Table 1). 

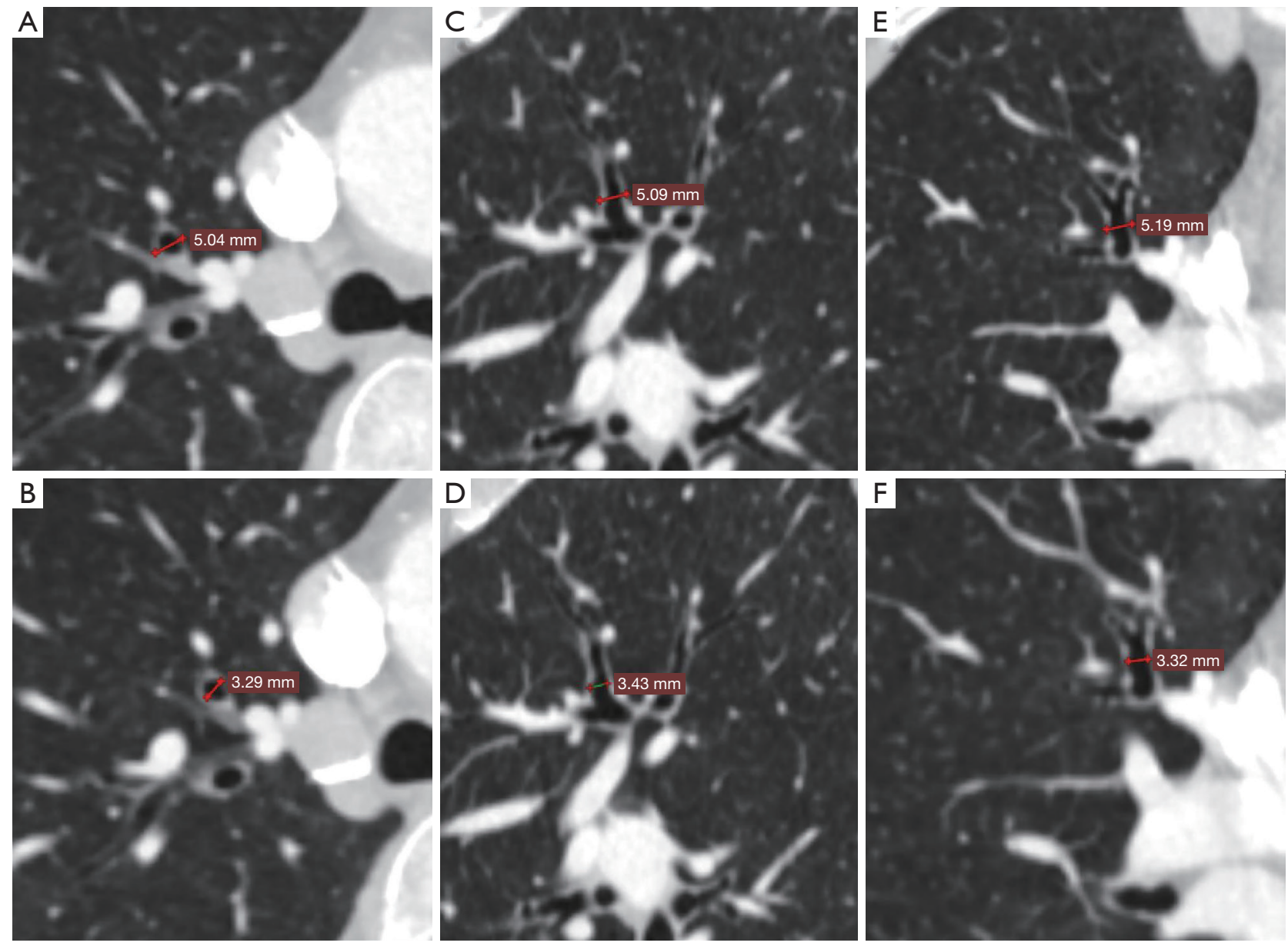

Figure 2 Measurement of the closed bronchial data on preoperative computed tomography (CT). (A,B) Top: bronchial outer diameter (BOD) of the apical segmental bronchus of the right upper lobe $\left(\mathrm{RB}^{1}\right)$ in the horizontal CT image; bottom: bronchial inner diameter (BID) of $\mathrm{RB}{ }^{1}$ in the horizontal CT image. (C,D) Top: BOD of $\mathrm{RB}^{1}$ in the sagittal CT image; bottom: BID of RB ${ }^{1}$ in the sagittal CT image. (E,F) Top: $\mathrm{BOD}$ of $\mathrm{RB}^{1}$ in the coronal CT image; bottom: BID of $\mathrm{RB}^{1}$ in the coronal CT image.

The diameter of targeted segmental bronchi was measured by preoperative CT image (Figure 2). The mean BOD was $6.02 \mathrm{~mm}$ and that of bronchial wall thickness was $0.86 \mathrm{~mm}$ in all cases of this study. We subtracted bronchial inner diameter (BID) from BOD, and then half of the numerical value was the bronchial wall thickness. There was no significant difference in bronchial wall thickness of resected bronchi between the two groups $(\mathrm{P}>0.05)$.

Segments distribution are shown in Table 2, 100 cases (61.7\%) underwent single segment resection, 48 cases (29.6\%) underwent combined segment resection, and 14 cases $(8.6 \%)$ underwent subsegment or combined subsegment resection. In subsegmentectomy, there were 12 cases in PVS group and 2 cases in PLC group, respectively.

There was a significant difference in blood loss between the two groups $(\mathrm{P}<0.05)$, with intraoperative hemorrhage in PVS group $(15.2 \pm 8.91 \mathrm{~mL})$ less than that in PLC group $(25.29 \pm 24.06 \mathrm{~mL})$. The operation time of PVS group was similar with PLC group $(\mathrm{P}>0.05)$. There was no significant difference between PVS group and PLC group in postoperative drainage and duration of drainage. The total incidence of postoperative complications was $14.8 \%$ (24/162), and there was no significant difference in hemoptysis between the two groups $(\mathrm{P}>0.05)$. No special treatment was needed to intervene the 24 patients who occurred hemoptysis for that the symptoms were all mild and intermittent. After 3 months of follow-up, the symptoms of postoperative hemoptysis all disappeared. The incidence of other postoperative complications including air leakage ( $>3$ days) (1 case) and subcutaneous emphysema 
Table 2 Segmentectomy position

\begin{tabular}{|c|c|c|c|c|}
\hline \multirow{2}{*}{$\begin{array}{l}\text { Segmentectomy } \\
\text { position }\end{array}$} & \multicolumn{2}{|c|}{ PVS } & \multicolumn{2}{|c|}{ PLC } \\
\hline & Left lung & Right lung & Left lung & Right lung \\
\hline Total number & 40 & 35 & 51 & 36 \\
\hline Segment & 36 & 27 & 51 & 34 \\
\hline$S^{1}$ & & 6 & & 8 \\
\hline$S^{2}$ & & 4 & & 5 \\
\hline$S^{1+2}$ & 19 & & 10 & \\
\hline$S^{1}+S^{2}$ & & 1 & & 2 \\
\hline$S^{3}$ & 1 & 3 & 1 & 5 \\
\hline$S^{1}+S^{3}$ & & 1 & & 2 \\
\hline$S^{1+2}+S^{3}$ & 5 & & 18 & \\
\hline$S^{1}+S^{6}$ & & & & 1 \\
\hline$S^{1+2}+S^{6}$ & & & 3 & \\
\hline$S^{4}+S^{5}$ & 3 & & 4 & \\
\hline$S^{3}+S^{4}+S^{5}$ & 1 & & 1 & \\
\hline$S^{5}+S^{6}$ & 1 & & & \\
\hline$S^{2}+S^{6}$ & & 2 & & \\
\hline$S^{6}$ & 2 & 4 & 11 & 10 \\
\hline$S^{8}$ & 2 & 1 & 2 & 1 \\
\hline$S^{6}+S^{8}$ & & 1 & & \\
\hline$S^{7}+S^{8}$ & 1 & 1 & & \\
\hline$S^{9}$ & & 2 & & \\
\hline$S^{10}$ & 1 & 1 & 1 & \\
\hline Subsegment & 4 & 8 & 0 & 2 \\
\hline S3a & & 1 & & \\
\hline $\mathrm{S} 3 \mathrm{~b}$ & & 2 & & \\
\hline$S^{8} b$ & 1 & & & \\
\hline$S^{6} b+S^{8} a$ & & & & 1 \\
\hline$S^{1}+S^{2} a$ & & & & 1 \\
\hline$S^{1+2} C$ & 1 & & & \\
\hline $\mathrm{S}^{1+2} \mathrm{C}+\mathrm{S}^{4} \mathrm{a}$ & 1 & & & \\
\hline $\mathrm{S}^{1+2} \mathrm{C}+\mathrm{S}^{3} \mathrm{a}$ & 1 & & & \\
\hline $\mathrm{S}^{1} \mathrm{a}+\mathrm{S}^{2} \mathrm{a}$ & & 2 & & \\
\hline$S^{1} b+S^{3}$ & & 1 & & \\
\hline$S^{2} b+S^{3} a$ & & 2 & & \\
\hline
\end{tabular}

PVS, powered vascular stapler; PLC, powered linier cutter.
(3 cases) were not enough for statistical analysis (Table 3).

All 28 surgeons compared the transection of segmental bronchi with PVS and PLC (Figure 3). For the subjective evaluation of PVS, $100 \%$ of the surgeons agree (slight or strongly agree) that PVS is easier to pass through the target segmental bronchus while reducing the number of attempts. Twenty surgeons (71.4\%) agree that PVS can reduce the concomitant injury on adjacent lung tissues or vessels. However, 8 surgeons (28.6\%) were neutral about whether the amount of dissection required was reduced and 6 surgeons $(21.4 \%)$ argue that PVS cannot reduce the amount of dissection required. There were still 10.7\% ( $n=3)$ of the surgeons who disagree the use of PVS can reduce the mental stress when anvil access to critical bronchi structures.

\section{Discussion}

Previous studies have shown that the occurrence of complications in lobectomy or segmentectomy is closely related to the closure of bronchial stump and vessels (9). The most serious complication of bronchial complications is bronchopleural fistula (BPF). However, the incidence of this complication in segmental resection (range, $0 \%$ to $0.3 \%$ ) is lower than that in lobectomy (range, $0.5 \%$ to $3.0 \%)(6,10,11)$. Kuroda et al. reported their experience with 217 patients underwent thoracoscopic segmentectomy, which subsegmental or segmental bronchus (SSB) was transected with a PVS in 73 (33.6\%) and observed no BPF formation (10). In the present study, there was also no complication of BPF formation.

Contrary to the study of Kuroda et al., there was no significant difference in BOD between PVS group and PLC group in our study. It allows us to assess the safety of PVS at the same baseline level. The average BOD in this study was $6.02 \mathrm{~mm}$ (5.91 and $6.11 \mathrm{~mm}$ in PVS and PLC group, respectively). This data was similar to the described segmental bronchial diameter, which were reported by Oizumi and Kuroda et al. $(6,10)$. The closed staple height of the PVS is $1.0 \mathrm{~mm}$, which means that the target segment bronchi should be compressed by thickness of $1.0 \mathrm{~mm}$. The mean thickness of the segmental bronchial wall measured by preoperative CT was $0.86 \mathrm{~mm}$, and the shape of nails after bronchial transection is good enough. Although the ideal thickness of the segmental bronchi wall is still unknown, PVS was safe for segmental bronchial transection in our study. 
Table 3 Intraoperative and postoperative data

\begin{tabular}{|c|c|c|c|}
\hline Variable & PVS & PLC & $P$ value \\
\hline Blood loss, $\mathrm{mL}$, mean \pm SD & $15.2 \pm 8.91$ & $25.29 \pm 24.06$ & 0.001 \\
\hline Postoperative drainage, $\mathrm{mL}$, mean $\pm \mathrm{SD}$ & $619.93 \pm 527.65$ & $715 \pm 462.41$ & 0.223 \\
\hline Chest tube duration, days, mean $\pm \mathrm{SD}$ & $4.33 \pm 1.70$ & $4.05 \pm 1.33$ & 0.23 \\
\hline Pulmonary air leakage ( $>3$ days) & - & 1 & - \\
\hline Subcutaneous emphysema & 2 & 1 & - \\
\hline
\end{tabular}

PVS, powered vascular stapler; PLC, powered linier cutter.



Figure 3 Results of subjective evaluation questionnaire. Q1: PVS is smoother than PLC in passing through the targeted segmental bronchi. Q2: The use of PVS can reduce the number of attempts of anvil through the segmental bronchus. Q3: Closure of segmental bronchi with PVS can reduce the concomitant injury on adjacent lung tissues or vessels. Q4: PVS can potentially reduce the amount of dissection required. Q5: The use of PVS can reduce the pressure of surgeon during the closure of the segmental bronchus. PVS, powered vascular stapler; PLC, powered linier cutter.

The main advantage of segmental bronchi closure using a PVS is the elaborate design, which makes it easier to placement on the segmental bronchi. The decrease in anvil perimeter is accomplished by changing from six rows of staples to four rows (12). In addition, the slim curved tip improves the posterior visibility of SSB and helps the surgeon to confirm the safe and accurate placement of the target bronchi. Another advantage of segmental bronchi transection with PVS is less intraoperative bleeding than that of PLC in our study. This difference has not been observed in the study of Kuroda et al. (10). Reduced intraoperative bleeding using PVS may be attributed to the fact that the tip with an additional $22^{\circ}$ of articulation helps to reduce the amount of dissection required before placement, while reducing the potential injury to fragile pulmonary tissues during transection. Further prospective studies are warranted to determine whether the use of PVS can effectively reduce intraoperative side effects in segmental bronchi transection.

Overall, the thoracic surgeons responded positively with respect to the use of PVS in segmental bronchi transection within medical practice. However, not all of them agreed that the use of PVS can reduce the amount of dissection required when passing through the target segmental bronchus. Nevertheless, in segmentectomy, a small but clinically significant impact on surgeons should not be neglected is the mental stress when anvil access to critical bronchi structures. Although this effect is negligible for senior surgeons, it undoubtedly enhances the confidence for younger surgeons in segmentectomy. Another interesting result in our study should be noticed is that the proportions of left upper division segment in PVS group (5/75) are significantly less than those in PLC group (18/87). One explanation could be that left upper division segment bronchus $\left(\mathrm{B}^{1+2}, \mathrm{~B}^{3}\right)$ is relatively thick. Therefore, as recommended by Kuroda et al., we agree that the use of a PVS for segmental bronchi that are compressed by less than $1.0 \mathrm{~mm}$.

In conclusion, we have objectively investigated the safety and efficacy of PVS on segmental bronchial transection. In the majority of segmentectomy, it is safe and feasible to use PVS instead of PLC for segmental bronchus closing. Further prospective studies are needed to accurately evaluate the application scope of PVS. At the same time, we look forward to more convenient and effective application of new stapler devices in thoracic surgery. 


\section{Acknowledgments}

Funding: The study was funded by Institutional Fundamental Research Funds (2018PT32033).

\section{Footnote}

Conflicts of Interest: The authors have no conflicts of interest to declare.

Ethical Statement: The authors are accountable for all aspects of the work in ensuring that questions related to the accuracy or integrity of any part of the work are appropriately investigated and resolved. This study was approved by the ethics committee of National Cancer Center/Cancer Hospital, Chinese Academy of Medical Sciences and Peking Union Medical College (approval number: 191137-1921). The requirement of patients' informed consent was waived owing to the retrospective nature of the study.

\section{References}

1. Hoy H, Lynch T, Beck M. Surgical Treatment of Lung Cancer. Crit Care Nurs Clin North Am 2019;31:303-13.

2. Dziedzic R, Zurek W, Marjanski T, et al. Stage I nonsmall-cell lung cancer: long-term results of lobectomy versus sublobar resection from the Polish National Lung Cancer Registry. Eur J Cardiothorac Surg 2017;52:363-9.

3. Aokage K, Yoshida J, Hishida T, et al. Limited resection for early-stage non-small cell lung cancer as functionpreserving radical surgery: a review. Jpn J Clin Oncol 2017;47:7-11.

Cite this article as: Ji Y, Qiu B, Gao S. The powered vascular staple (PVS) versus conventional powered linier cutter (PLC) for the application of bronchial transection in thoracoscopic anatomic segmentectomy. J Thorac Dis 2019;11(11):4647-4653. doi: $10.21037 /$ jtd.2019.10.68
4. Yamanashi K, Okumura N, Otsuki Y, et al. Stapler-Based Thoracoscopic Basilar Segmentectomy. Ann Thorac Surg 2017;104:e399-402.

5. Sato M, Murayama T, Nakajima J. Concepts and techniques: how to determine and identify the appropriate target segment in anatomical pulmonary segmentectomy? J Thorac Dis 2019;11:972-86.

6. Oizumi H, Kato H, Endoh M, et al. Management of Bronchial Stumps in Anatomic Lung Segmentectomy. Ann Thorac Surg 2016;101:2120-4.

7. Kuroda H, Yoshida T, Sakao Y. A powered vascular staple for the application of segmental bronchial closure in thoracoscopic anatomic segmentectomy. J Thorac Dis 2017;9:5352-4.

8. Wang J, Xu X, Wen W, et al. Technique for tailoring complex demarcation in lung segmentectomy. Thorac Cancer 2018;9:1562-4.

9. Brioude G, Gust L, Thomas PA, et al. Postoperative complications after major lung resection. Rev Mal Respir 2019;36:720-37.

10. Kuroda H, Sugita Y, Nakanishi K, et al. Favorable clinical application for segmental bronchial closure based on experiment results. J Thorac Dis 2019;11:2267-73.

11. Okada M, Tsutani Y, Ikeda T, et al. Radical hybrid videoassisted thoracic segmentectomy: long-term results of minimally invasive anatomical sublobar resection for treating lung cancer. Interact Cardiovasc Thorac Surg 2012;14:5-11.

12. Ng CS, Pickens A, Siegel JM, et al. A novel narrow profile articulating powered vascular stapler provides superior access and haemostasis equivalent to conventional devicesdagger. Eur J Cardiothorac Surg 2016;49 Suppl 1:i73-8. 